\title{
I Einleitung*
}

\section{Problemaufriss}

In Deutschland gibt es keinen Wohnungsmangel per se, sondern eine heterogene Entwicklung der Nachfrage. So verschärft sich der „Run“ auf Wohnraum insbesondere in sogenannten „Schwarmstädten“, während es andernorts Leerstände gibt. Bei den „Schwarmstädten“ handelt es sich um jene Orte, in denen nicht bloß ein Bevölkerungswachstum zu verzeichnen ist, sondern dieses durch Binnenwanderung erzeugt wird und zwar vor allem durch jüngere Altersgruppen. ${ }^{1}$ Jene Städte zeichnen sich also insbesondere durch eine besondere Attraktivität für jüngere Altersgruppen im bundesweiten Vergleich aus. ${ }^{2}$ Dabei treten sich selbst verstärkende Effekte hinzu, da gerade die Anwesenheit junger Menschen die Attraktivität für diese Zielgruppe erhöht. ${ }^{3}$ Entsprechend heterogen entwickeln sich in Deutschland die Mietpreise, ${ }^{4}$ vor allem auch abhängig von sich extrem heterogen entwickelnden Baulandpreisen. ${ }^{5}$

\footnotetext{
* Der Beitrag präsentiert Ergebnisse, die der Verfasser u.a. im Rahmen eines Forschungsaufenthalts als Visiting Scholar an der UC Berkeley erarbeitet hat. Dies gilt insbesondere für die ökonomischen Hintergründe der Bekämpfung der Gentrifizierung im Rahmen der Mietenregulierung. Teile der vorliegenden Ausführungen sind bereits veröffentlicht worden und zwar zur Kompetenzfrage Kühling Länderkompetenz für „Mietendeckel“? - Keine Kakophonie in der Mietpreisregulierung!, DVBl. 2020, 842, und zu materiell-rechtlichen Fragen der Mietenregulierung mit Blick auf Art. 14 GG Kühling Gentrifizierung als Rechtsproblem - Wohnungspolitik ohne rechtsstaatliche Leitplanken?, NZM 2020, 521. Der Verfasser betreut inzwischen eine Verfassungsbeschwerde gegen die Berliner Mietpreisregelung von zwei Vermietern vor dem Bundesverfassungsgericht. Der Text wurde gegenüber dem am 12.2.2020 vor der Juristischen Gesellschaft zu Berlin gehaltenen Vortrag deutlich erweitert und punktuell aktualisiert.

1 Vgl. Simons/Weiden Schwarmverhalten, Reurbanisierung und Suburbanisierung, Information zur Raumentwicklung, BBSR Heft 3.2016, S. 263, abrufbar im WWW unter der URL https://d-nb.in fo/1155025490/34 (zuletzt abgerufen am 1.7.2020).

2 Vgl. zur Problematik Simons Sicherung der Wohnraumversorgung in Deutschland, in: ifo Schnelldienst 21/2018, S. 12ff., abrufbar im WWW unter der URL https://www.cesifo.org/DocDL/ sd-2018-21-11-8.pdf (zuletzt abgerufen am 1.7.2020).

3 Simons/Weiden Schwarmverhalten, Reurbanisierung und Suburbanisierung, Information zur Raumentwicklung, BBSR 3/2016, S. 273, abrufbar im WWW unter der https://d-nb.info/ 1155025490/34 (zuletzt abgerufen am 1.7.2020), die davon sprechen, dass das Schwarmverhalten vom „Wunsch nach Zusammenrottung - also die unzureichende Dichte junger Menschen als Folge des Geburteneinbruchs auszugleichen“ getrieben wird.

4 Siehe etwa unter Hinweis auf den Zusammenhang von Wanderungseffekten und Mietenentwicklung für den Zeitraum von 2011 bis 2016 Mense gif Policy Paper 2/2019, Angebotseffekte der
}

๑ OpenAccess. (c) 2020 Juergen Kuehling, publiziert von De Gruyter. (cc) BY-NC-ND Dieses Werk ist lizenziert unter der Creative Commons Attribution-NonCommercial-NoDerivatives 4.0 License.

https://doi.org/10.1515/9783110719031-001 


\section{Die Situation in Berlin im Vergleich zu anderen „Schwarmstädten“}

Die Entwicklung der Mietpreise ist in Deutschland dementsprechend ebenfalls äußerst heterogen. Dabei gehört Berlin, was die Mietpreishöhe selbst bei den deutlich höheren Neuvermietungsverträgen anbelangt, längst noch nicht zu den teuersten Städten Deutschlands. ${ }^{6}$ Unter den sieben besonders attraktiven deutschen Großstädten (Berlin, Düsseldorf, Frankfurt am Main, Hamburg, Köln, München, Stuttgart) ist Berlin im Jahr 2019 nach wie vor die Stadt mit der günstigsten mittleren Angebotsmiete. ${ }^{7}$ Allerdings ist die Preisentwicklung angesichts jahrelang moderater und teils rückläufiger Entwicklungen der Miethöhen in den letzten Jahren sehr stark ansteigend gewesen. Es sind also Nachholeffekte aufgetreten. So sind die Miethöhen im Neuvermietungsmarkt in der vergangenen Dekade in Berlin am stärksten, nämlich um $60 \%$ gestiegen, ${ }^{8}$ allerdings ausgehend von einem ungewöhnlich niedrigen Preisniveau für eine attraktive Großstadt in Deutschland und erst recht im Vergleich zu anderen attraktiven Hauptstädten in Europa.

Im Übrigen zeigt die Mietpreisentwicklung in Berlin ein differenziertes Bild. So ist vor allem zu unterscheiden zwischen der Preisentwicklung in Bestandsmietverhältnissen und der in Neuvermietungsverhältnissen. Die Mietpreise in Bestandsmietverhältnissen sind im Zeitraum der vergangenen 20 Jahre vor Beginn der Diskussion um den „Mietendeckel“ in Berlin, namentlich von 1999 bis 2018,

Mietpreisbremse, S. 1f., abrufbar im WWW unter der URL https://www.gif-ev.de/onlineshop/de tail/440 (zuletzt abgerufen am 1.7.2020).

5 Die Untersuchung für den Zeitraum von 2011 bis 2017 von Prognos Endbericht „Wer baut in Deutschland?“ - Studie zum Wohnungsbautag 2019, S. 11f., abrufbar im WWW unter der URL https://www.prognos.com/uploads/tx_atwpubdb/20190509_Inventur_zum_Bauen_und_Woh nen_2019_-_PROGNOS-Wohnungsbau-Studie.pdf (zuletzt abgerufen am 1.7.2020), weist Unterschiede von einer fast erfolgten Verdopplung der Baulandpreise in den sieben größten Städten Deutschlands Berlin, Düsseldorf, Frankfurt, Hamburg, Köln, München und Stuttgart und weiteren attraktiven Universitätsstädten wie Freiburg, Münster oder Regensburg aus. In einigen attraktiven Oberzentren stiegen die Preise um 50\%, während sie im Schnitt nur um etwa ein Drittel stiegen und in einer ganzen Reihe von ausgeglichenen Märkten um gerade einmal 10\%.

6 Siehe die Hinweise bei Zeit-online, abrufbar im WWW unter der URL https://www.zeit.de/wirt schaft/2019-11/mietpreise-steigerung-mietspiegel-wohnungsnot-gemeinden (zuletzt abgerufen am 1.7.2020).

7 Simons/Schmandt Frühjahresgutachten. Wohnimmobilien 2020, S. 178, abrufbar im WWW unter der URL https://zia-cloud.de/data/public/d5a531 (zuletzt abgerufen am 1.7.2020).

8 Simons/Schmandt aaO. 
jährlich um 2,8\% bzw. um 0,14 €/qm gestiegen. ${ }^{9}$ Das ist zwar höher als die durchschnittliche Inflationsrate in diesem Zeitraum, wird aber für den Durchschnitt der Wohnbevölkerung mehr als relativiert angesichts der günstigen Lohnentwicklungen. So sind die mittleren Bruttoarbeitsentgelte in den Jahren von 2013 bis 2018 um 15,3\% gestiegen, ${ }^{10}$ was in etwa der Entwicklung der Bestandsmieten im selben Zeitraum entspricht. Für den Durchschnitt der Mieter und damit für breite Bevölkerungsgruppen hat sich die Wohnungssituation also keineswegs verschlechtert. Probleme treten hingegen bei Bestandsmietern für jenen deutlich geringeren Personenkreis auf, der von der günstigen Entwicklung der Löhne nicht profitiert hat.

Deutlich spürbarer sind im Vergleich jedoch die Preiserhöhungen in Neuvertragsmietverhältnissen. Hier haben sich die Durchschnittspreise bedeutend stärker erhöht, so dass für einen größeren Kreis von Personen der Zugang zu bezahlbarem Wohnraum nach einem Wohnungswechsel in Berlin oder Zuzug nach Berlin problematisch geworden ist. So betrug der Preisunterschied Anfang 2019 zwischen Bestands- und Neuvertragsmieten in Berlin fast 27 Prozentpunkte. ${ }^{11}$ Diese schon jetzt große Diskrepanz zwischen Bestands- und Neuvertragsmieten in Berlin ist ein Grund dafür, dass die Umzugsquoten in Berlin deutlich unterhalb der bundesdeutschen Umzugsquoten liegen und in den Jahren von 2013 bis 2017 nochmals stark gesunken sind, namentlich von 7,6 auf 5,9\%. ${ }^{12}$ Es ist davon auszugehen, dass daher der sogenannte „Mismatch“, d.h. das Verharren der Mieter in an sich ungünstigen Wohnungen, in Berlin besonders hoch ist. Dies ist für die Verfügbarkeit von Wohnraum besonders für die zahlreichen Fälle problematisch, in denen der Wohnraum deutlich zu groß ist, aber ein Wechsel aus einer günstigen großen Wohnung aus einem Bestandsmietverhältnis in eine teure kleine Wohnung im Rahmen einer Neuvertragsmiete nicht sinnvoll ist. Damit werden die zu großen Wohnungen nicht für passende Nachfrager (etwa Familien) frei. Allerdings ist auch insoweit zu differenzieren und zwar sowohl hinsichtlich der verschiedenen Stadtteile Berlins als auch in Bezug auf die unterschiedlichen

9 Mieterschutzbund Berlin, Berliner Mietspiegel 2019 in Kraft - Grund zur Freude, 03/2019, S. 10, abrufbar im WWW unter der URL https://www.mieterschutzbund-berlin.de/mieterschutz-2019. html?file=tl_files/content/Mieterschutz/2019/3/Mieterschutz_03_2019.pdf (zuletzt abgerufen am 1.7.2020).

10 Siehe dazu die Hinweise bei Sagner/Voigtländer Volkswirtschaftliche Folgen des Berliner Mietendeckels, Gutachten im Auftrag der CDU-Fraktion Berlin, November 2019, S. 11, abrufbar im WWW unter der URL https://www.iwkoeln.de/fileadmin/user_upload/Studien/Gutachten/PDF/ 2019/IW-Gutachten_2019-Mietendeckel.pdf, (zuletzt abgerufen am 1.7.2020).

11 Sagner/Voigtländer aaO, S. 10.

12 Sagner/Voigtländer aaO, S. 9. 
Marktsegmente: So sinken die Mieten bereits seit 2017 in besonders einfachen Lagen in Berlin, beispielsweise in Charlottenburg-Nord, Marzahn oder Neu-Hohenschönhausen, während sie in besonders beliebten und guten Lagen, etwa in Dahlem, Mitte, Prenzlauer Berg oder Tiergarten auch im Jahr 2019 weiter angestiegen sind. ${ }^{13}$ Insgesamt sind jedenfalls die Angebotsmieten seit dem 2. Quartal 2018 im Schnitt gesunken, wenn auch nur geringfügig um 0,7\%. Betrachtet man diesen Preis unter Abzug der allgemeinen Preissteigerung von 1,4\%, sind die Angebotsmieten im Jahr 2018 real um 2,1\% gesunken. ${ }^{14}$ In welchem Umfang dies auf die vom Bundesgesetzgeber eingeführte „Mietpreisbremse“ zurückgeht, ist unklar. Eindeutig ist jedoch die Trendumkehr in Richtung durchschnittlich leicht sinkender Angebotsmieten. Allerdings zeichnet sich schon jetzt ab, dass der radikale Berliner „Mietendeckel“ einen erheblichen Preissteigerungsdruck auf den prozentual geringen Bereich der regulatorisch noch nicht erfassten Neubauten im Rahmen der Neuvermietung ausgelöst hat und in diesem Marktsegment die Mieten in den ersten Monaten nach Inkrafttreten der Berliner Regelung gestiegen sind. ${ }^{15}$

In der breiten Masse der Bestandsmietverhältnisse ist damit selbst in Berlin eine moderate Mietpreisentwicklung erfolgt, die in etwa der Lohnentwicklung entspricht und daher in der Breite nicht zu einer Verschlechterung des Zugangs zu bezahlbarem Wohnraum geführt hat. Probleme treten hingegen bei der Entwicklung der Neuvertragsmieten auf, die jedoch selbst in Berlin kein flächiges Problem mehr darstellt.

\section{Breite der Diskussion und Fokus der folgenden Untersuchung}

Die steigenden Mieten führen zu weiteren Konsequenzen, die politisch teils unerwünscht sind. Allen voran gilt dies für die sogenannte Gentrifizierung. Darunter

13 Simons/Schmandt Frühjahresgutachten. Wohnimmobilien 2020, S. 181, abrufbar im WWW unter der URL https://zia-cloud.de/data/public/d5a531 (zuletzt abgerufen am 1.7.2020).

14 Simons/Schmandt aaO, S. 179.

15 Damit verstärkt sich der schon durch die „Mietpreisbremse“ im BGB vorgezeichnete Trend, siehe die Nachweise, dass im Jahreszeitraum von Mai 2019 bis Mai 2020 die Neuvermietungen für regulierte Objekte um knapp 2\% von durchschnittlich 12,90€ auf 12,66€ pro Quadratmeter gefallen sind, während die Mieten für Neubauten mit Baujahr nach 2014 im selben Zeitraum um $7,5 \%$ von durchschnittlich 16,92€ auf 18,19€ pro Quadratmeter gestiegen sind, abrufbar im WWW unter der URL https://embed.presseportal.de/de/31321/article/4625790 (zuletzt abgerufen am 1.7. 2020). 
ist die schleichende Verdrängung der bisherigen Wohnbevölkerung in signifikantem Umfang durch zahlungskräftigeres Publikum zu verstehen. ${ }^{16}$ Die Vielzahl von Handlungsinstrumenten ausgehend vom klassischen Mieterschutzrecht ergänzt um die „Mietpreisbremse“ über teils punktuelle, teils flächige Zweckentfremdungs- und Milieuschutzsatzungen ${ }^{17}$ bis hin zur jüngsten Diskussion um Baugebote ${ }^{18}$ oder Enteignungen von Wohnungsbaugesellschaften ${ }^{19}$ deuten auf die Vielgestaltigkeit und Komplexität des Themas aus rechtlicher und ökonomischer Sicht hin. Jegliche rechtliche Bewertung, die Aussagen zur Wirkungsweise der Instrumente verlangt, wie insbesondere ihre Verhältnismäßigkeitsprüfung im Rahmen einer Prüfung am Maßstab der Eigentumsfreiheit, bedarf einer ökonomischen Fundierung. Diese soll daher in einem ersten Schritt ganz allgemein mit Blick auf die Besonderheiten der Wohnungsmärkte erfolgen (dazu II.). Angesichts der mittlerweile breiten Diskussion der vielfältigen Handlungsinstrumente und ihres tatsächlichen oder vermeintlichen Beitrags zur Lösung der wohnungspolitischen Probleme ist für die Strukturierung der Debatte sodann deren Klassifikation erforderlich, die kombiniert werden soll mit einer knappen ökonomischen Wirkungsanalyse (dazu III.). Jene ökonomische Analyse der Wirkungszusammenhänge ist im Weiteren - wie aufgezeigt - relevant für die verfassungsrechtliche Bewertung der Handlungsinstrumente. Diese soll mit besonderem Blick auf die jüngst eingeführten und erwogenen Vorschläge zur Mietenregulierung vertieft werden, einerseits in Bezug auf die grundlegende Fragen des Schutzes der sozialen Marktwirtschaft aufwerfende eigentumsrechtliche Debatte (dazu IV.) und andererseits hinsichtlich des auf fundamentale Gerechtigkeitsfragen stoßenden gleichheitsrechtlichen Diskurses (dazu V.). Verfassungsrechtlich fundierte Verfahren sind dabei in unteren Instanzen teils erfolgreich gewesen, ${ }^{20}$ während die

16 Zum Begriff Schulz Aufwertung und Verdrängung in Berlin - Räumliche Analysen zur Messung von Gentrifizierung, in: Statistisches Bundesamt, WISTA, 04/2017, S. 62, abrufbar im WWW unter der URL https://www.destatis.de/DE/Methoden/WISTA-Wirtschaft-und-Statistik/2017/04/ aufwertung-verdraengung-berlin-042017.pdf?_blob=publicationFile (zuletzt abgerufen am 1.7. 2020).

17 So hat Berlin aktuell 57 Milieuschutzgebiete, vgl. z. B. Behnke/Dammann GE 2019, 431 (434).

18 Dazu Köster BauR 2019, 1378; siehe auch Bunzel/Niemeyer ZfBR 2018, 743 (748ff.).

19 Dies als verfassungswidrig ablehnend Kloepfer NJW 2019, 1656; Schmidt DÖV 2019, 508.

20 Hinsichtlich der verfahrensrechtlichen Anforderungen im Rahmen der Begründung gemäß $\S 556$ d Abs. 2 S. 5 u. 6 BGB BayVerfGH, Beschl. v. 4.4.2017 - Vf. 3-VII-16, ZfIR 2017, 509 (LS); LG Frankfurt, Urt. v. 27.3.2018 -2-11 S 183/17, WuM 2018, 276 (LS); LG Hamburg, Urt. v. 14. 6. 2018-333 S 28/17, WuM 2018, 498 (LS); zu Begründungsdefiziten und zur daraus folgenden Nichtigkeit der Hessischen Mietenbegrenzungsverordnung von 2015 BGH, Urt. v. 17.7.2019 - VII ZR 130/18, NJW 2019, 2844; LG Berlin, Urt. v. 10.10. 2019-65 S 107/19, NJW 2019, 3730 (LS), lässt hingegen bereits eine Begründung an anderer Stelle als im Gesetz- und Verordnungsblatt ausreichen; siehe hin- 
mit Spannung erwartete Entscheidung des BVerfG über die Verfassungsbeschwerde gegen die bundesweite „Mietpreisbremse“ und die Berliner Mietenbegrenzungsverordnung erfolglos blieb. ${ }^{21}$ Die Analyse des Beschlusses ist allerdings sehr aufschlussreich für die weitere Bewertung der diversen wohnungspolitischen Handlungsvorschläge. Schließlich war die Frage, ob die Länder neben dem Bund überhaupt Mietpreisregelungen erlassen dürfen, von Anfang an einer der großen verfassungsrechtlichen Streitpunkte der Diskussion um einen „Mietendeckel“ in Berlin. So sind während des Berliner Gesetzgebungsprozesses eine Reihe von Gutachten veröffentlicht worden ${ }^{22}$, die sich mit dieser scheinbar „nüchternen“ Kompetenzfrage auseinandersetzen, die angesichts der politischen Brisanz der Mietenregulierung besonders delikat ist. Neben dem Verfassungsgerichtshof des Landes Berlin wird sich auch das BVerfG mit dieser Frage befassen müssen. So wurde nicht nur ein abstraktes Normenkontrollverfahren auf Bundesebene frühzeitig angekündigt ${ }^{23}$ und schließlich auch eingereicht ${ }^{24}$, vielmehr hat das Landgericht Berlin mit Beschluss vom 12. März 2020 das Gesetz im Rahmen einer konkreten Normenkontrolle auf den Karlsruher Prüfstand gestellt. Das Landgericht Berlin geht davon aus, dass dem Land Berlin keine Gesetzgebungskompetenz für die Mietpreisregulierung zusteht. ${ }^{25}$ Da auch in Bayern ein Volksbegehren über einen „Mietenstopp“ initiiert worden ist ${ }^{26}$, zeigt sich die allgemeine Bedeu-

sichtlich eines Verstoßes gegen Art. 3 GG die weitere Überlegung des LG Berlin, Beschl. v. 14.9. 2017-67 S 149/17, NZM 2017, 766 und LG Berlin, Vorlagebeschl. v. 7.12.2017-67 S 218/17, NJW 2018, 728.

21 BVerfG, Beschl. v. 18.7.2019-1 BvL 1/18, abrufbar im WWW unter der URL http://www.bverfg. de/e/lk20190718_1bvl000118.html - ECLI:DE:BVerfG:2019:1k20190718.1bvl000118. (zuletzt abgerufen am 1.7.2020)

22 Siehe dazu die Nachweise in den folgenden Fn. 109, 134, 142, 147 und 153.

23 Siehe etwa den Bericht in der FAZ online, abrufbar im WWW unter der URL https://www.faz. net/aktuell/wirtschaft/berliner-mietendeckel-soll-vom-verfassungsgericht-geprueft-werden16639904.html (zuletzt abgerufen am 1.7.2020).

24 Siehe u. a. den Bericht im Handelsblatt online, abrufbar im WWW unter der URL https://www. handelsblatt.com/politik/deutschland/normenkontrollklage-union-und-fdp-wollen-mietende ckel-in-berlin-kippen/25805072.html (zuletzt abgerufen am 1.7.2020); auf Landesebene haben Abgeordnete der CDU- und FDP-Fraktion im Abgeordnetenhaus eine abstrakte Normenkontrolle beim Berliner Verfassungsgerichtshof eingereicht, vgl. dazu den Bericht im Tagesspiegel, abrufbar im WWW unter der URL https://www.tagesspiegel.de/berlin/berliner-verfassungsgerichtshofcdu-und-fdp-klagen-gegen-mietendeckel/25857444.html (zuletzt abgerufen am 1.7.2020).

25 LG Berlin, Beschl. v. 12.3.2020 - 67 S 274/19, NZM 2020, 368.

26 Der Gesetzesentwurf findet sich u.a. auf der Homepage der Initiatoren im WWW unter der URL https://mietenstopp.de/wp-content/uploads/2019/10/191021_volksbegehren-mietenstopp-ge setzestext.pdf (zuletzt abgerufen am 1.7.2020); inzwischen hat der Bayerische Verfassungsgerichtshof entschieden, dass das Volksbegehren aufgrund einer sogar offensichtlich fehlenden 
tung, die kompetenzrechtliche Frage zu klären, ob die Länder - parallel zum Bund - Mietpreisregelungen erlassen dürfen (dazu VI.).

Landeskompetenz unzulässig ist, siehe BayVerfGH, Entsch. v. 16.7.2020 - Vf. 32-IX-20, BeckRS 2020, 16071; gegen diese Entscheidung hat die Initiative wiederum Verfassungsbeschwerde eingelegt, offenbar wegen eines angeblichen Verstoßes gegen das Recht auf den gesetzlichen Richter, siehe dazu u. a. den Bericht in der SZ, abrufbar im WWW unter der URL https://www.sueddeut sche.de/bayern/muenchen-volksbegehren-mietenstopp-verfassungsgericht-1.5002604 (zuletzt abgerufen am 20.9.2020) 